\title{
EINSATZ EINES TERRESTRISCHEN 3-D-LASERSCANNERS IN EPHESOS
}

Im Jahr 2002 wurde in Ephesos erstmals ein 3-D-Laserscanner für die Dokumentation archäologischer Strukturen verwendet (Abb. 1). Der Einsatz dieser modernen Technologie erfolgte im Rahmen des Projekts $»$ Die Topographie der Oberstadt von Ephesos« des Österreichischen Archäologischen Instituts durch VIAS - Vienna Institute for Archaeological Science in Kooperation mit der Firma RIEGL Laser Measurement Systems, Horn, wobei die Anwendungsbereiche dieser neuen Technologie in der archäologischen Feldforschung untersucht und erprobt werden sollten ${ }^{1}$.

Die 3-D-Lasersensoren der Firma RIEGL LMS ermöglichen die Erfassung dreidimensionaler Information beliebiger Oberflächen mit hoher Geschwindigkeit und Auflösung in einem Abstand von 1$1000 \mathrm{~m}$ und in einem Sichtfeld von $90 \times$ $360^{\circ}$. Die Messung basiert auf Ermittlung der Laufzeit von Infrarot-Impulsen, kombiniert mit einer opto-mechanischen Scanvorrichtung. Diese Technologie liefert dichte und sehr genaue Punktwolken

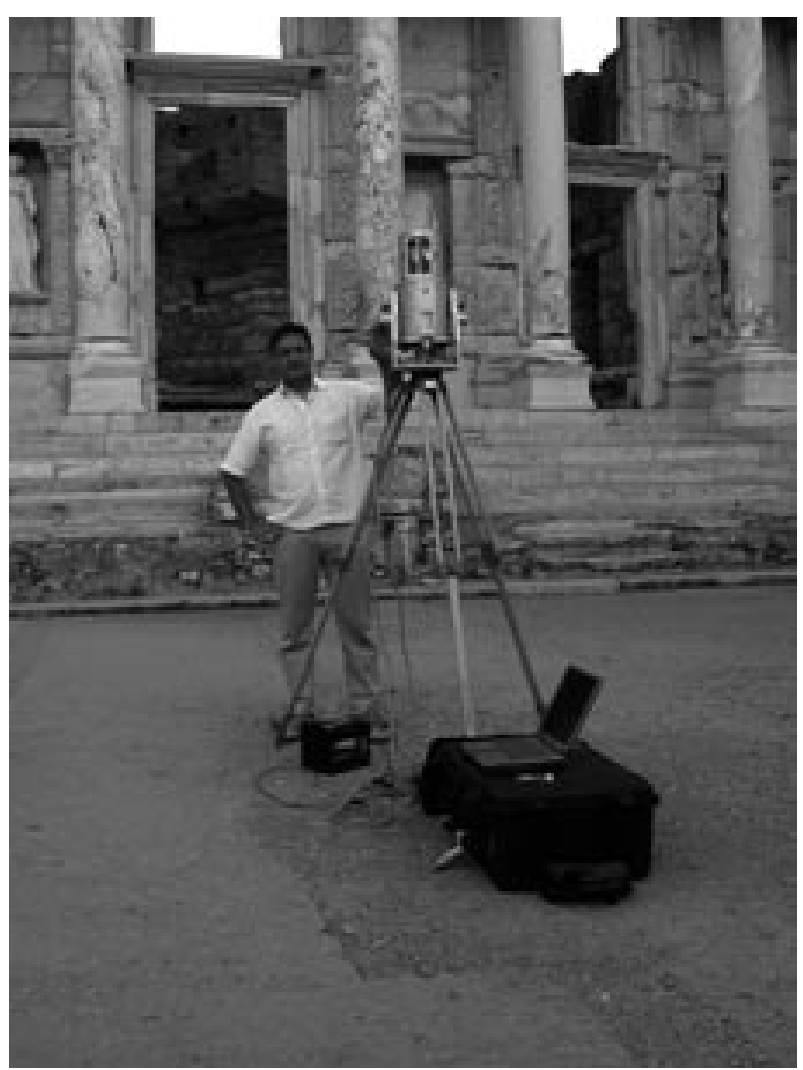

1 3-D-Laserscanner RIEGL LMS-Z210 im Einsatz vor der Celsusbibliothek in Ephesos, Herbst 2002 der gescannten Oberflächen, zusätzlich werden Information zur Reflektivität (Reflexionsintensität) sowie ein RGB-Farbwert zu jedem gescannten Datenpunkt erfaßt, die direkt für eine Texturierung der aus den Punktwolken generierten 3-D-Datenmodelle genutzt werden können. Die Anwendungsbereiche in der Archäologie ${ }^{2}$ liegen in der hochauflösenden Erfassung von Architekturteilen und historischen Gebäuden ${ }^{3}$, Monumentalstatuen, Grabkammern, Höhlen etc. wie auch in der Erfassung topographischer Daten einer Fundstelle bis zur Dokumentation einzelner Schichtoberflächen bei stratigraphischen Grabungen. Die gewonnenen Daten können entweder zur momentanen Dokumentation, vergleichbar einer photogrammetrischen Aufnahme, dienen oder als Grundlage für die Erstellung eines virtuellen Modells der Fundstelle weiterverarbeitet werden.

1 Besonderer Dank für ihre Unterstützung soll N. Studnicka und A. Ullrich von der Fa. RIEGL Laser Measurements Systems ausgesprochen werden.

2 A. Ullrich - N. Studnicka - J. Riegl - W. Neubauer, 3D laser-sensors and their application in Archaeology and modeling of historic buildings, Archaeological Prospection 2004 (in Druck).

3 M. Afshar - M. Boroumand - N. Studnicka, Archaeological Scanning of Persepolis. Using 3D laser scanning for mapping archaeological sites in Iran, Geomatics International Magazine 6, 16, 2002, 1-5. 


\section{Methode, archäologische Voraussetzungen und Ziele}

Im Rahmen des Forschungsprojekts »Die Topographie der Oberstadt von Ephesos « wurde eine systematische und flächendeckende Gelände- und archäologische Bestandsaufnahme der Oberstadt von Ephesos, d. h. von den Ausläufern der Straße nach Magnesia bis zum sog. Staatsmarkt bzw. zur Unteren Agora, mit Hilfe einer Real Time Kinematik (RTK)-Geo Positioning System (GPS)-Ausrüstung vorgenommen ${ }^{4}$; eines der Ergebnisse dieser Surveys war ein hochauflösendes digitales Geländemodell (Abb. 2).

Die Methode der Datenaufnahme im Feld besteht darin, das Areal möglichst flächendeckend zu begehen und Geländepunkte dreidimensional, mit einer Genauigkeit im Zentimeterbereich, einzumessen. Die Meßpunkte werden bei einer Aufnahme im steilen Gelände selektiv auf Bruchkanten, in ebenem Gelände hingegen flächig in einen Raster gesetzt. Die Auswahl der Meßpunkte vor Ort in Abhängigkeit von der topographischen Situation ist für die hohe Qualität des Geländemodells ausschlaggebend; bei der Auswertung hochauflösender Luftbilder ist diese in der

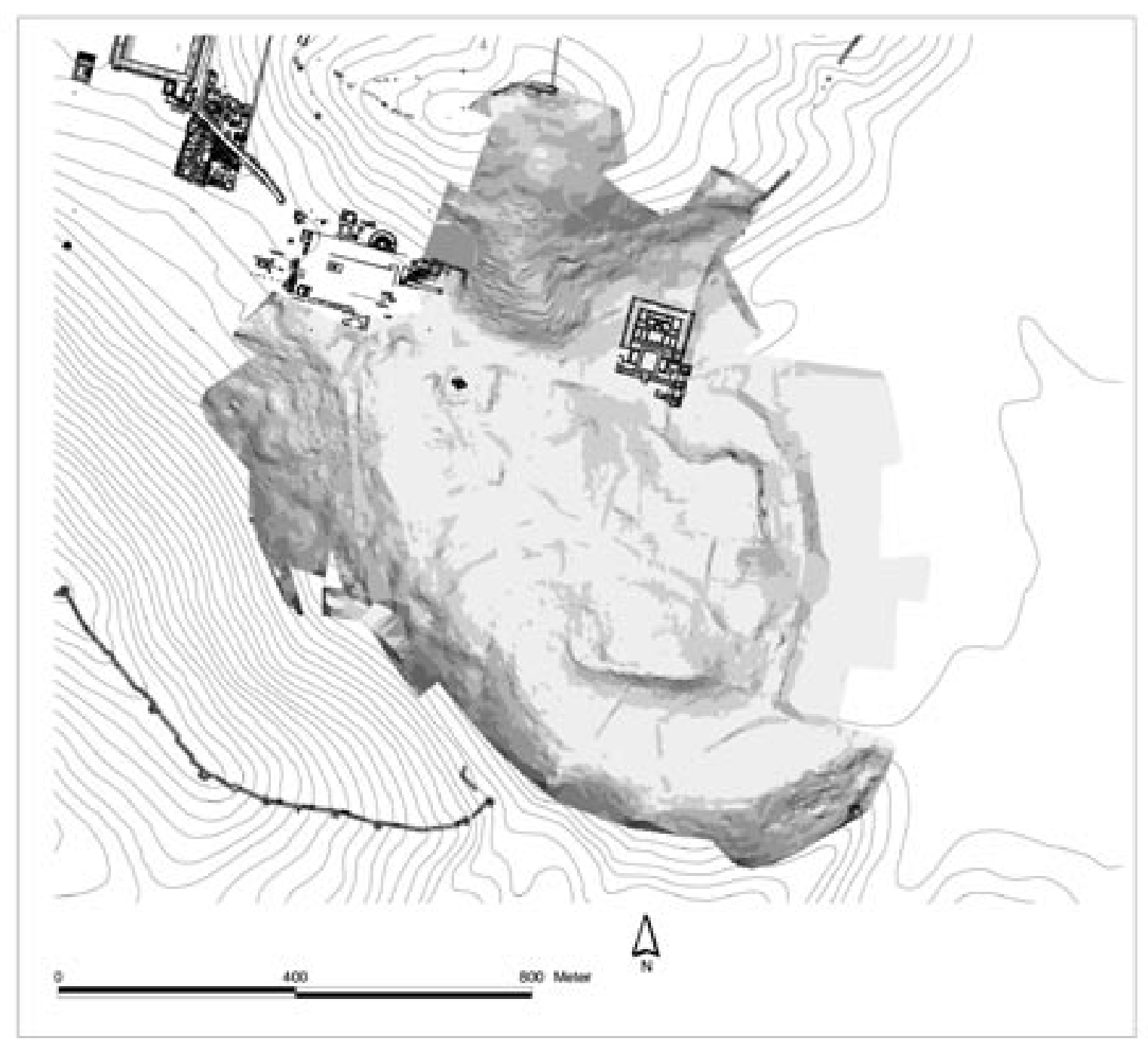

2 Digitales Geländemodell der Oberstadt von Ephesos, berechnet aus der GPS-Aufnahme

${ }^{4}$ St. Groh, Die Topographie der Oberstadt von Ephesos. Vorbericht über das Projektjahr 2000, ÖJh 70, 2001, 21-33. 


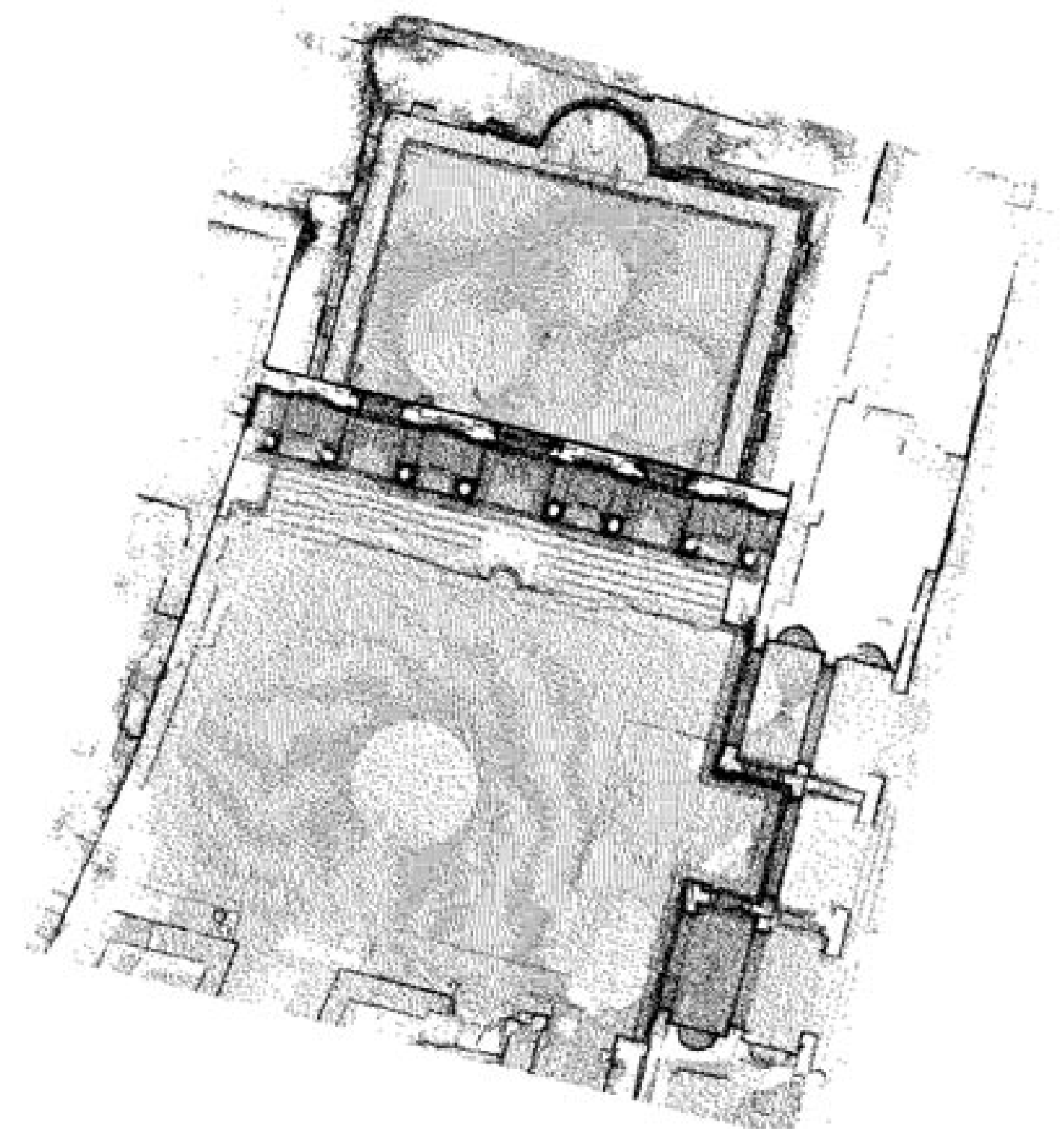

3 Senkrechte Draufsicht auf die Punktwolke der Celsusbibliothek, zusammengesetzt aus 19 Scans und reduziert auf einen regelmäßigen Raster von $40 \times 40 \times 40 \mathrm{~cm}$

Form nicht zu erreichen. Die Daten der archäologischen Bestandsaufnahme fließen in ein Geoinformationssystem (GIS; in diesem Fall: ArcView 8 von ESRI) ein, wo sie mit den Auswertungen von Luftbildern, den Daten der geophysikalischen Prospektionen und den archäologischen Zusatzinformationen (Befund-, Bild- und Literaturdatenbanken) verwaltet und analysiert werden. Diese Methode des intensiven Surveys ist jedoch relativ zeitaufwendig. Für die Aufnahme eines steilen Geländes von 12 ha Fläche benötigt ein Team von zwei Personen ungefähr zwei Arbeitswochen.

Ein konkret zu testender Anwendungsbereich für den 3-D-Laserscanner stellt die topographische Aufnahme einer archäologischen Landschaft dar. In Ephesos galt es zu untersuchen, wie sich die Datenqualität der 3-D-Laserscans zu derjenigen der Luftbildauswertung und des Surveys verhält, wie sich der z. T. doch recht dichte Bewuchs auf das zu generierende Geländemodell auswirkt und für welche Fragestellung welcher Methode der Vorzug zu geben ist. Als Testareal wurde das Gelände um die Obere Agora, der sog. Staatsmarkt, gewählt. Die archäologischen Anforderungen an den Laserscan waren: eine hochauflösende Punktwolke des Geländes der 


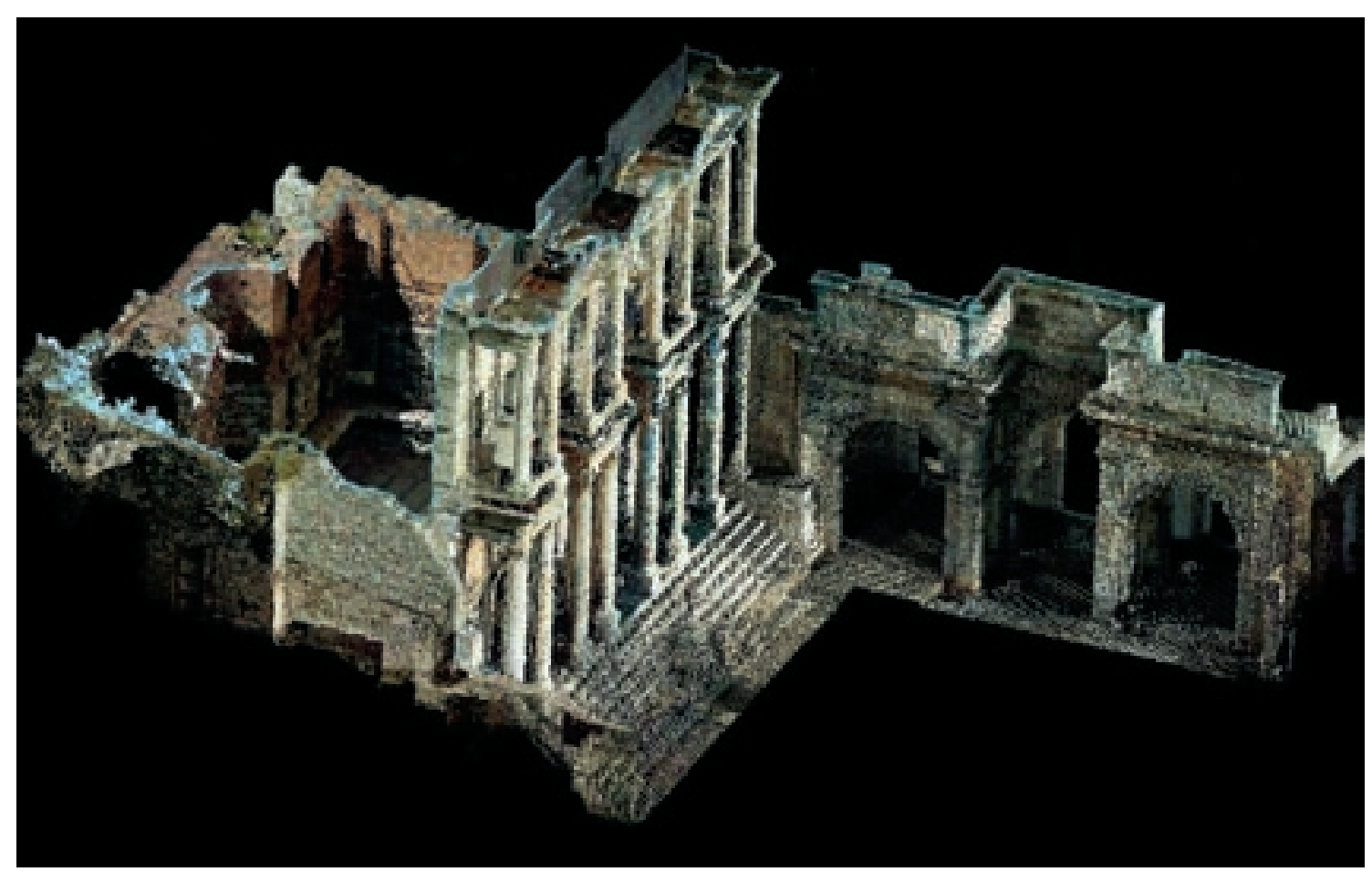

4 Schrägansicht von Südosten eines ausgeschnitten Bereichs der Punktwolke mit Farbwerten, reduziert auf einen regelmäßigen Raster von $5 \times 5 \times 5 \mathrm{~cm}$

Agora und der sie umgebenden steilen Hänge (Südhänge des Panayir Dağ und Nordhänge des Bülbül Dağ) als Ergänzung des bereits bis zum Ostrand der Agora generierten digitalen Geländemodells, eine detaillierte Punktwolke von den die Agora umgebenden antiken Bauten, speziell des Bouleuterions/Odeions, und die Implementierung dieser großen Datenmengen in das GIS, insbesondere in das 3-D-Modell der Oberstadt.

Als weitere Einsatzmöglichkeit dieser Geräte für die zukünftige archäologische Forschung in Ephesos sollte die dreidimensionale Vermessung komplexer antiker Baukörper bzw. von Fassaden getestet werden. Als Anwendungsbeispiel dafür wurde der für Ephesos wohl bekannteste Bau, die 1978 wiedererrichtete Ostfassade der im ersten Viertel des 2. Jahrhunderts n. Chr. erbauten Celsusbibliothek ausgewählt. Die Anforderung an den Laserscan war, eine möglichst detailgetreue Aufnahme der Fassade und ihrer Umgebung mit einer Auflösung im Zentimeterbereich für eine dreidimensionale Visualisierung (Abb. 3. 4) zu erreichen. Ein zusätzliches, für kommerzielle Anwendungen nicht zu unterschätzendes Produkt dieser Laserscans sollte ein Glaswürfel sein, in den die Daten maßstabgetreu eingebrannt werden können. Anhand dieses Modells, das in jedem beliebigen Maßstab erzeugt werden kann, ist es möglich, den Bau von jeder Seite aus uneingeschränkt zu betrachten.

Gleichzeitig sollten durch die Testmessungen auch die logistischen Bedingungen für einen systematischen Einsatz dieser Technologie in Ephesos geklärt werden; für den Testeinsatz standen fünf Arbeitstage zur Verfügung. Bereits bei der ersten Begehung wurde eines der Hauptprobleme offensichtlich: Touristenströme verhinderten eine Durchführung der Arbeiten zum günstigsten Zeitpunkt, nämlich bei möglichst hochstehender Sonne und kurzem Schlagschatten. Es mußte daher mit den frühen Morgenstunden Vorlieb genommen werden. 


\section{Meßgerät und Arbeitsablauf}

Bei dem 2002 eingesetzten Meßgerät handelt es sich um einen RIEGL LMS-Z210 3-D-Laserscanner. Das $13 \mathrm{~kg}$ schwere Gerät scannt einen maximalen horizontalen Bereich von $335^{\circ}$ und einen vertikalen Bereich von $80^{\circ}$ mit einer maximalen Auflösung von 0.08 gon. Ein Scanvorgang dauert je nach gewählter Auflösung zwischen 20 Sekunden und 4 Minuten. Die Genauigkeit der Distanzmessung liegt bei Distanzen unter $350 \mathrm{~m}$ bei $25 \mathrm{~mm}$, bei Distanzen bis $700 \mathrm{~m}$ bei $50 \mathrm{~mm}$. Die räumliche Auflösung des Geräts wird durch die Genauigkeit der Distanzmessung und die Divergenz des Meßstrahls von 3 mrad bestimmt. Das Instrument eignet sich bei archäologischen Anwendungen gut für Distanzen von 3-150 m, wobei seine kompakte und robuste Bauweise eine wesentliche Voraussetzung für den archäologischen Einsatz ist.

Der Laserscanner mißt die Distanz zu einem Zielpunkt und gleichzeitig den entsprechenden Vertikal- und Horizontalwinkel. Im Gegensatz zu einer Totalstation muß der Meßstrahl nicht auf einen Zielpunkt ausgerichtet werden, sondern mißt in einem regelmäßigen polaren Raster im Sichtfeld des Instruments. Das Gerät erfaßt bei jedem Meßvorgang gleichzeitig Distanz, Reflexionsintensität und RGB-Farbwert $(3 \times 16$ bit). Vereinfacht gesagt, werden in einem Meßdurchgang die Geometrie wie auch ein digitales Farbbild der gescannten Umgebung erfaßt; die Qualität des Farbbildes hängt dabei wesentlich von der Ausleuchtung der gescannten Oberflächen ab.

Der primäre binäre Datenstrom besteht aus einer dreidimensionalen Punktwolke (X, Y, Z) mit den beiden Attributen Reflektivität und Farbwert im relativen Koordinatensystem des Instruments. Da von einem einzelnen Aufstellungsort nicht alle Bereiche einsichtig sind und das verwendete Gerät einen toten Winkel von $25^{\circ}$ aufweist, sind meist mehrere Positionierungen notwendig, um dreidimensionale Objekte vollständig zu erfassen. Dadurch wird es notwendig, die einzelnen Scans in ein gemeinsames Koordinatensystem zu transformieren. Dieser Vorgang, als Registrierung bezeichnet, wird normalerweise über stark reflektierende Paßpunkte vorgenom-

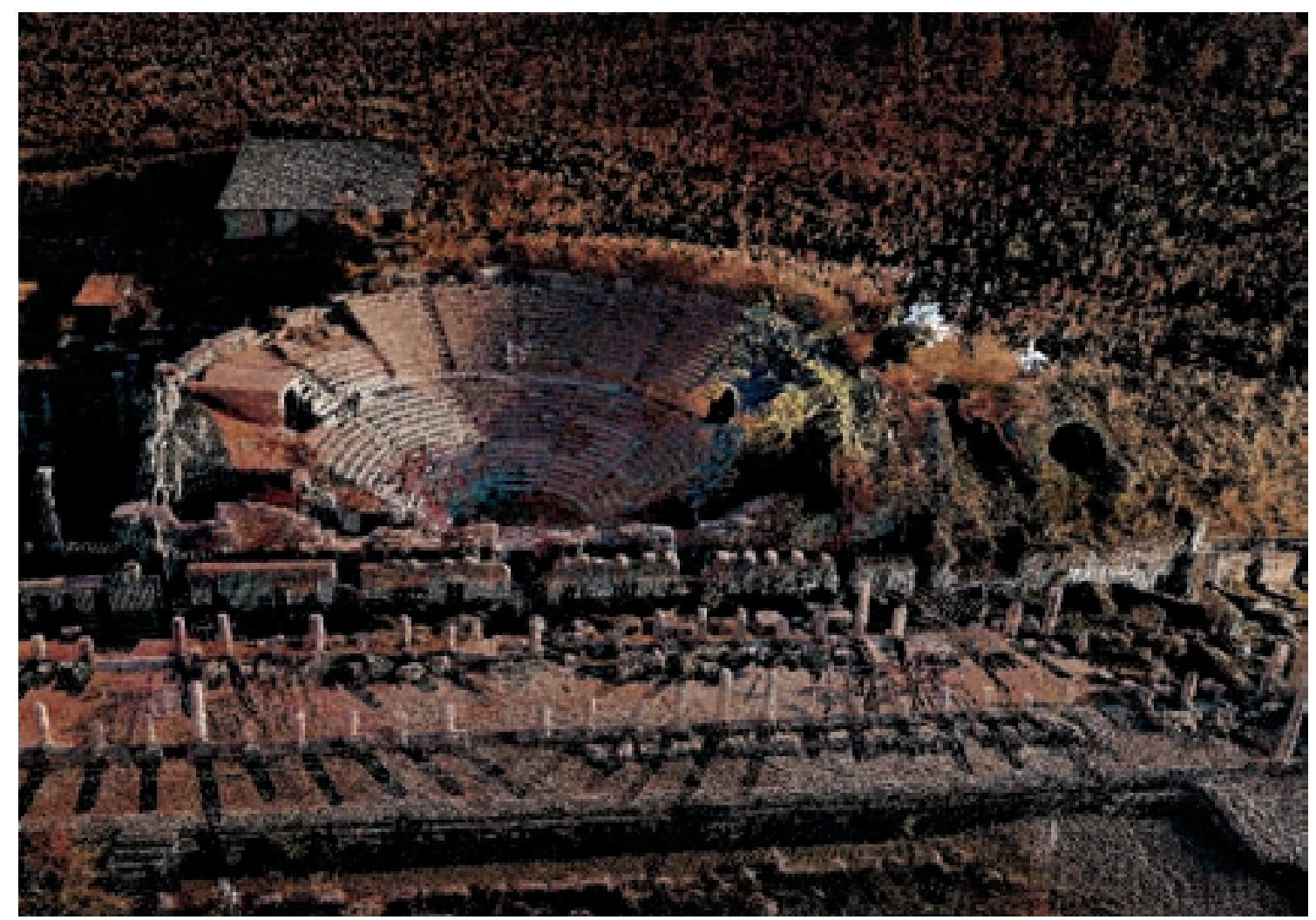

5 Ausschnitt aus dem dreidimensionalen Modell des 'Staatsmarktes' und des 'Odeions'. Blick von Westen 
men, wobei jeweils mindestens vier Paßpunkte in zwei aufeinanderfolgenden Scans sichtbar sein müssen. Werden diese Paßpunkte beispielsweise mit einer Totalstation in absoluten Koordinaten eingemessen, können die einzelnen Scans bei der Registrierung direkt in das absolute Koordinatensystem transformiert werden. Die bei dem Meßvorgang aufgezeichnete Intensität des reflektierten Signals ist gut für eine automatische Objekterkennung geeignet und ermöglicht es, u. a. die reflektierenden Paßpunktmarken in den Daten automatisch zu erkennen.

Für die vollständige Erfassung der Fassade und des Innenraums der Celsusbibliothek und des Südtors der Unteren Agora wurden 19 Scans angefertigt (Abb. 3. 4. 11). Eine Arbeitsbühne auf dem Südtor wurde errichtet, nach der Verteilung der Paßpunktmarker wurde jeder Teilbereich von verschiedenen Standorten aus gescannt, wobei ein angeschlossener Laptop, auf dem auch die Daten abgespeichert werden, den Scanner steuerte. Die gescannten Farbbilder und die Intensitätsbilder bieten eine gute Kontrollmöglichkeit für eine erste Überprüfung des Scans: Detailbereiche können ausgewählt und gesondert gescannt werden. Aufgrund der bereits ab $8 \mathrm{Uhr}$ morgens nach Ephesos strömenden Touristen war es nicht möglich, länger als bis $9.30 \mathrm{Uhr}$ zu scannen, so daß möglichst schnell von einem Standort zum anderen gewechselt werden mußte und nur Zeit für einen 'Rundum-Scan' blieb. Nach Abschluß jeweils eines Teilbereichs wurden die Paßpunkte mit einer Totalstation absolut eingemessen, wobei auch bei der Verteilung der Paßpunktmarker in Ephesos darauf zu achten ist, sie in Positionen aufzustellen, die für Touristen möglichst unerreichbar sind, will man sie nicht als Souvenir in aller Herren Länder wissen. Dies erscheint lapidar, soll aber zeigen, welcherart die größten Probleme bei der Dokumentation der Celsusbibliothek waren, denn das Gerät selbst läßt sich leicht in allen gewünschten Positionen aufstellen, arbeitet auch in heißem Klima und staubiger Umgebung robust und zuverlässig.

Im Bereich des 'Staatsmarktes' wurden 10 Scans aufgenommen, wobei darauf geachtet wurde, besonders im Bereich des Bouleuterions/Odeions vollständige Aufnahmen zu erreichen (Abb. 5); durch zusätzliche Aufnahmen könnte die Datenqualität noch deutlich verbessert werden. Die Gesamtzeit für die Datenerfassung inklusive aller Auf- und Abbauarbeiten betrug sieben Stunden. In dieser Zeit konnten brauchbare auszuwertende Geländedaten (8 277106 Meßpunkte) für eine Fläche von 20 ha gewonnen werden.

\section{Ergebnisse und Schlußfolgerungen}

\section{Obere Agora, sog. Staatsmarkt}

Bei der Erstellung eines topographischen Modells von Ephesos ergeben sich bezüglich des zu erreichenden Ergebnisses zwei extreme Positionen: Einerseits kann das Ergebnis eine möglichst detaillierte Oberfläche aller vorhanden Strukturen sein - dem entsprechen bereits die Rohdaten der Scans -, wodurch Bäume, Sträucher, Mauern, Säulen etc. im Modell erhalten bleiben; man erhält also ein realistisches Abbild der Oberfläche. Auf der anderen Seite steht eine von allen Störungen gereinigte und idealisierte Geländeoberfläche, wie sie bei einem GPS-Survey, durch Auswahl der Meßpunkte und definierte Bruchlinien, leicht erreicht werden kann. Bei der Auswertung von Luftaufnahmen kann dies durch interaktive Auswahl der Meßpunkte oder durch eine an eine automatische Messung aus dem Stereomodell anschließende Korrektur einzelner Bereiche oder Meßpunkte im Modell vorgenommen werden. Die hohe Meßpunktdichte der Scandaten, in diesem Fall durchschnittlich 40 Punkte, im zentralen Bereich des gescannten Areals mehrere tausend pro Quadratmeter, erlaubt solche manuellen Korrekturen nicht mehr. Bei der weiteren Verarbeitung im GIS stellen solche Datenmengen ebenfalls ein Problem dar und müssen reduziert werden. Bei der GPS-Aufnahme (Abb. 2) wurden auf 120 ha durchschnittlich 0,015 Meßpunkte pro Quadratmeter, d. h. ca. alle $66 \mathrm{~m}^{2}$ ein Punkt, aufgenommen.

Um ein topographisches Modell zu erhalten, wurden für die bisherige Auswertung der Daten die Programme 3D-RiSCAN und RiCube verwendet. Ziel der Verarbeitung war, ein möglichst getreues Modell der Geländeoberfläche im GIS zu generieren. Die Daten wurden im ASCII- 

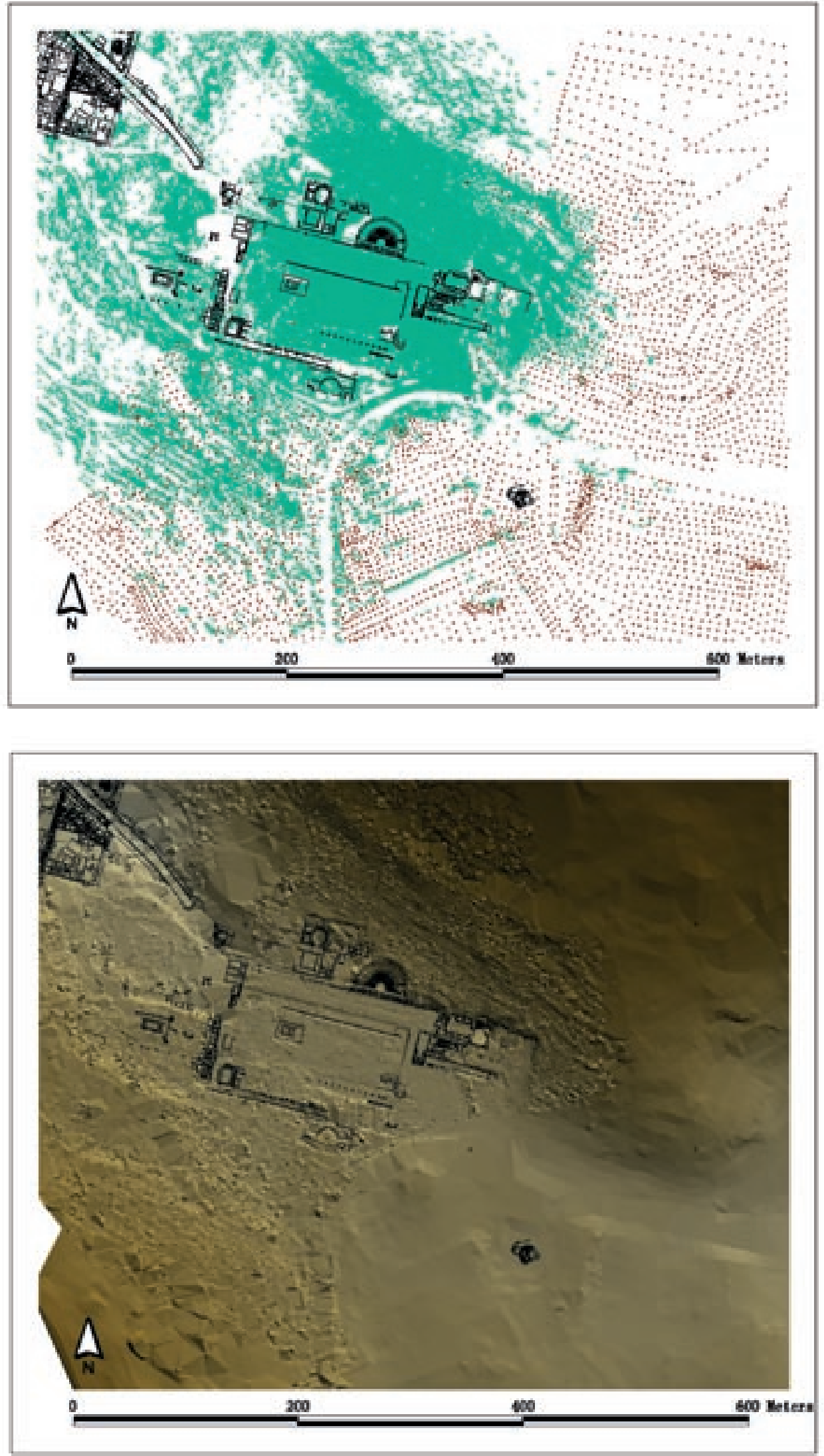

6 oben: Vergleich der Punktdichte des digitalen Geländemodells der GPS-Aufnahme und der Laserscans (reduziert auf einen Raster von $1 \times 1 \mathrm{~m}$ ); unten: Vergleich des triangulierten Geländemodells der GPS-Aufnahme und der 3-D-Laserscanner-Aufnahme 
Format exportiert, editiert und als 'Generate Format' in das Programm ArcView importiert. Die Visualisierung der Punktwolke stellte dabei kein Problem dar, die Triangulation der über 8 Millionen Punkte ist jedoch nicht zielführend. Die Daten wurden daher für die weitere Verarbeitung im GIS auf einen regelmäßigen Raster von $1 \times 1 \times 20 \mathrm{~m}$ großen Quadern reduziert $(150837$ Punkte). Mit RiCube ist es möglich, alle Meßwerte innerhalb eines solchen Quaders auf den tiefsten auftretenden Wert zu reduzieren. Dadurch kann bereits ein Großteil der störenden Vegetation ausgefiltert werden, Nachteil ist aber, daß auch viele Mauern und Säulen ausgefiltert werden. Die Daten wurden dadurch auf 86720 Meßpunkte reduziert (Abb. 6).

Vergleicht man das triangulierte Ergebnis (Abb. 6) mit dem Geländemodell, welches mit GPS erstellt wurde, erkennt man deutlich eine höhere Rauheit im Bereich der Berghänge. Eine manuelle Korrektur offensichtlicher Fehler ist möglich, aber äußerst arbeitsintensiv. Reduziert man die Scandaten auf die Auflösung des GPS-Surveys (3 789 Punkte), erweisen sich die Daten als sehr gut vergleichbar. Durch geringe manuelle Korrekturen und eine Extraktion der Bruchkanten aus den Scandaten könnte so bereits ein direkt vergleichbares Ergebnis erzielt werden, obwohl die Qualität des GPS-Surveys durch die kontrollierte Positionierung der Meßpunkte natürlich höher ist. Eine weitere Möglichkeit aus den Scannerdaten ein geglättetes Geländemodell zu erhalten, könnte durch gleitende Mittelwert- oder Medianfilter mit Zusatzbedingungen erreicht werden. Diese Verfahren sind zwar für die Erstellung eines generellen Geländemodells, vergleichbar einer Luftbildauswertung mit ausgesprochen hoher Punktdichte, einsetzbar, in Bereichen mit Mauerstrukturen oder ausgeprägten Geländeformationen zerstören sie jedoch die Vorteile des 3-D-Laserscans; sie können also nur selektiv eingesetzt werden. Ein entsprechendes Softwaretool sollte daher eine selektive Bearbeitung einzelner Ausschnitte aus dem Geländemodell mit unterschiedlichen Verfahren ermöglichen. Der große Vorteil des Laserscanners, die schnelle Datenaufnahme und die hohe Punktdichte, kann durch eine langwierige Nachbearbeitung wieder aufgehoben werden. Es ist aber zu berücksichtigen, daß die ausgewählte Testfläche in Ephesos ein für die GPS-Aufnahme nur schwer zugängliches Gebiet darstellt und viele Details wie z. B. Felswände nur durch berührungslose Messung erfaßt werden können. Ebenso läßt sich die Qualität der Scanneraufnahme durch weitere Aufstellungsorte noch beträchtlich erhöhen und damit auch eine leichtere Entfernung der Vegetation aus den Daten erreichen.

Für eine Bestandsaufnahme einer archäologischen Landschaft ist ein zeitintensiver, flächiger Survey unumgänglich, denn nur durch einen engen Begehungsraster sind Oberflächenfunde und Architekturteile zu dokumentieren. Die Verwendung eines GPS und eines GIS zur Lokalisierung und Archivierung der Funde erlaubt, im selben Arbeitsgang auch ein Geländemodell zu erfassen. Im vorliegenden Fall besitzt die Scanneraufnahme im Durchschnitt eine um das 2 666fache größere Punktdichte: Gebüsch und Bewuchs stören die Aufnahme der Topographie und können nur mit weiterem Aufwand adäquat zum Ergebnis der Begehung und der GPS-Aufnahme weggefiltert werden. Für einen Laserscan benötigt man jedoch nur einen Bruchteil der Zeit, die für einen Survey oder die photogrammetrische Auswertung eines Luftbildes aufzuwenden ist. Für eine grobe Geländeaufnahme und eine detailgetreue Implementierung einzelner archäologischer Bauwerke in ein GIS oder dreidimensionales Modell einer antiken Stadt bzw. einer Landschaft kann die Auflösung eines bereits an Daten reduzierten Scans als ausreichend und gut geeignet betrachtet werden, die Qualität ist deutlich höher als die einer photogrammetrischen Luftbildauswertung (Abb. 5). Ein Problem für die Erstellung eines Laserscans in bekannten archäologischen Zonen sind die Besucher, die sich innerhalb der Meßbereiche befinden und später nur schwer aus den Aufnahmen zu filtern sind; das bedingt, daß meist nur während Randzeiten gemessen werden kann: Tageslicht bzw. Beleuchtung der Topographie oder einzelner Bauwerke sind dann für eine qualitätvolle RGB-Farbinformation entsprechend schlecht geeignet. Es wäre jedoch durchaus möglich, unter Verzicht auf die Farbinformation die Topographie auch nachts aufzunehmen. 


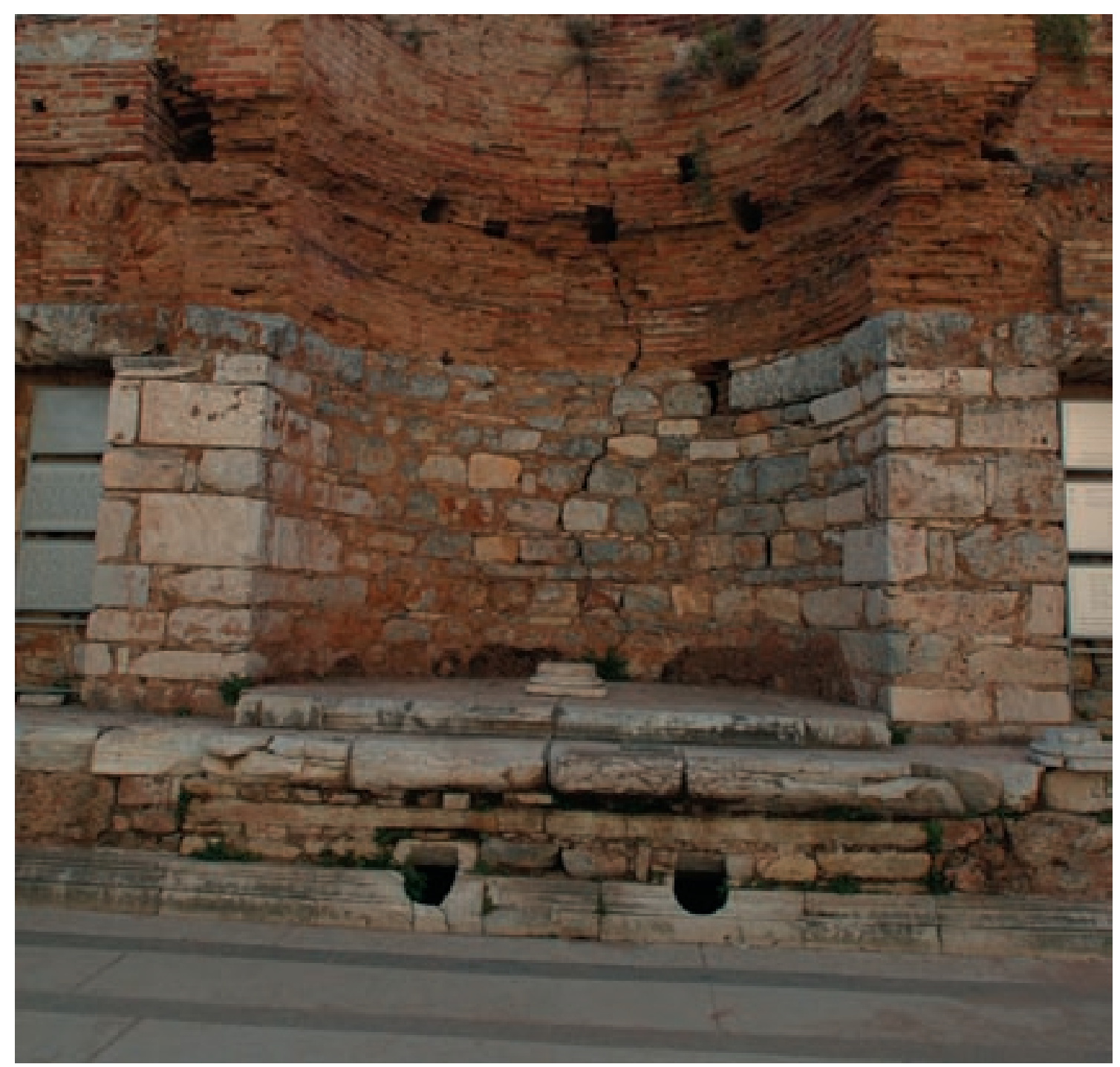

7 Digitales Photo der zentralen Apsis in der Celsusbibliothek
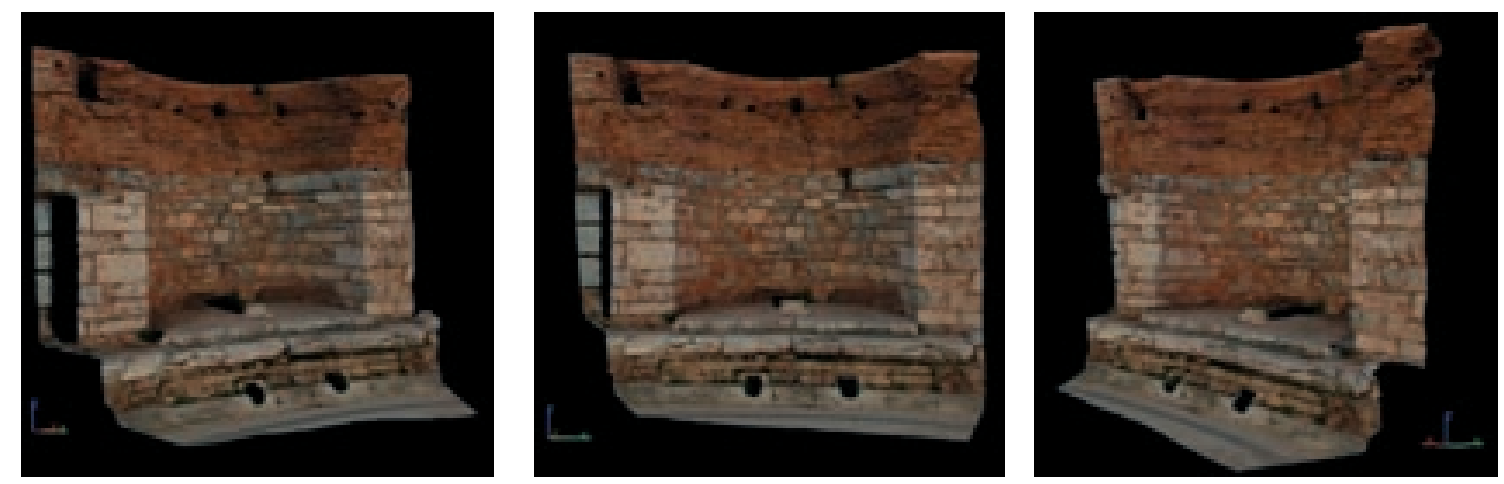

8 Drei Ansichten des 3-D-Modells der zentralen Apsis der Celsusbiblitohek, beruhend auf einem einzigen Scan 


\section{Celsusbibliothek}

Die Dokumentation der Celsusbibliothek gestaltete sich - wie erwähnt - vor allem durch den Besucherandrang ausgesprochen schwierig. Abgesehen von diesen Schwierigkeiten, die bei der Auswertung dazu führten, daß nur einzelne Bereiche an sich guter Scans verwendet werden konnten, hat sich das Gerät bewährt. Die Qualität der meisten aufgenommenen Farbbilder ist jedoch äußerst gering, Schatten und unterschiedliche Beleuchtung beeinträchtigen den optischen Gesamteindruck. Für eine qualitativ ansprechende 3-D-Farbdarstellung wäre eine detaillierte digitale photographische Dokumentation anzuschließen, um diese Bilder über Paßpunkte auf das geometrische Modell zu übertragen. Umgekehrt lassen sich von beliebigen Photos nun hochgenaue Orthophotos auf Grundlage der 3-D-Daten erstellen, die sodann für weitere Auswertungen zur Verfügung stehen. Aus den hochauflösenden geometrischen 3-D-Daten sind auch beliebige horizontale oder vertikale Profile abzugreifen, die für eine Weiterverarbeitung in ein CAD-Programm exportiert werden können.

Die zentrale Apsis im Innenraum der Bibliothek wurde exemplarisch dargestellt (Abb. 7-10). Die registrierten Rohdaten eines einzelnen Scans wurden in drei Ansichten dargestellt (Abb. 8), wobei die Punktdichte im Bereich von unter $1 \mathrm{~cm}$ liegt und sich durch die Kombination mehrerer Scans weiter erhöht. Aus den kombinierten Daten lassen sich beliebige Detailbereiche ausschneiden und weiterverarbeiten, aber bereits ein einzelner Scan (Abb. 8) bietet eine ausreichend genaue Grundlage für eine dreidimensionale, steingerechte Auswertung, etwa mit Hilfe einer

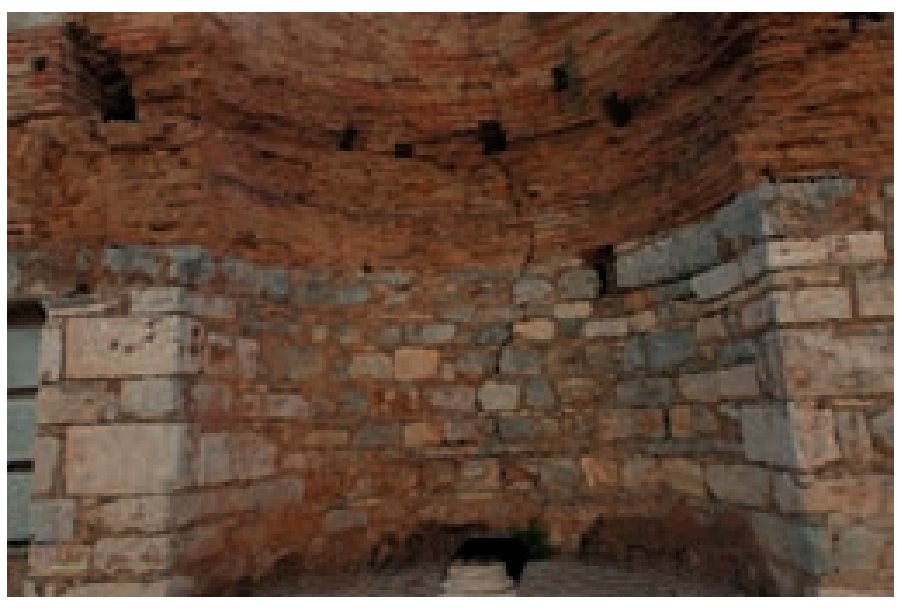

9 Zentrale Apsis der Celsusbibliothek, vergrößerte 3-D-Ansicht eines einzigen Scans photogrammetrischen Software. Die für die Testauswertung verwendete Software 'Reconstructor' von 3-D-Veritas ermöglicht es, neben der 3-D-Darstellung der Meßdaten auch eine 3-D-Triangulation der Meßdaten vorzunehmen (Abb. 10). Auf dieses triangulierte Modell können hochauflösende Photos kartiert werden, so daß ein realistisches 3-D-Modell für eine anschließende Animationen erzeugt werden kann.

Der 3-D-Laserscanner bietet die Möglichkeit einer raschen und detailgenauen Dokumentation von
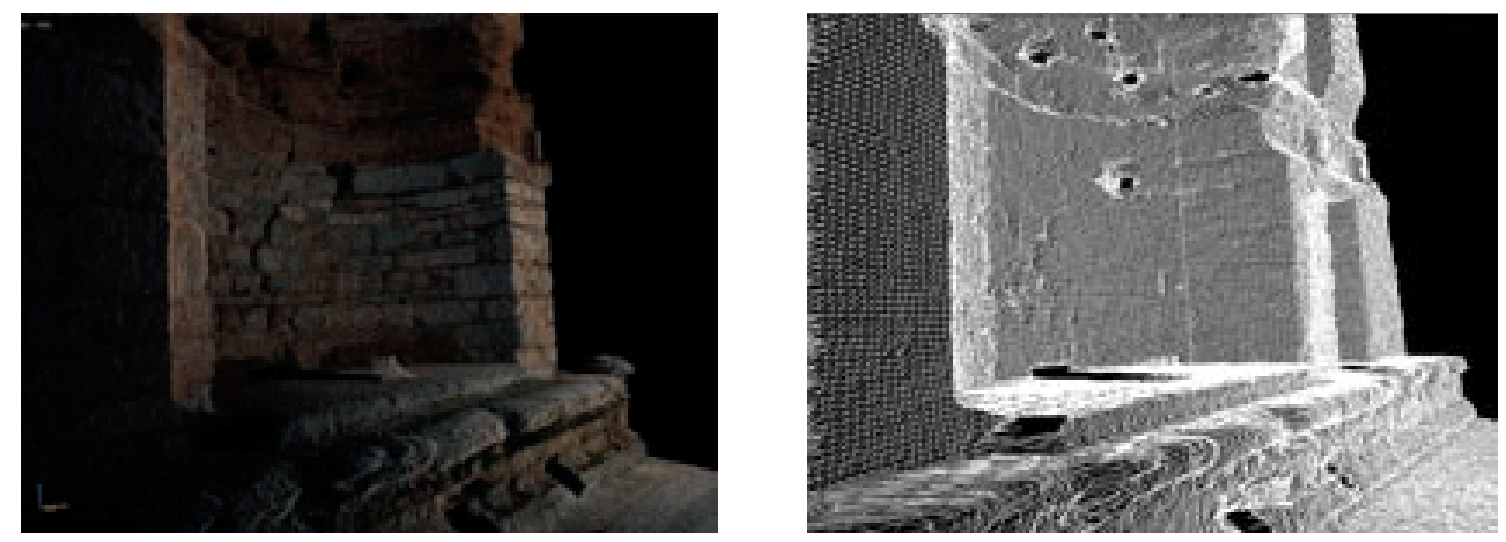

10 links: 3-D-Punktwolke eines einzelnen Scans; rechts: triangulierte Punktwolke desselben Ausschnitts 


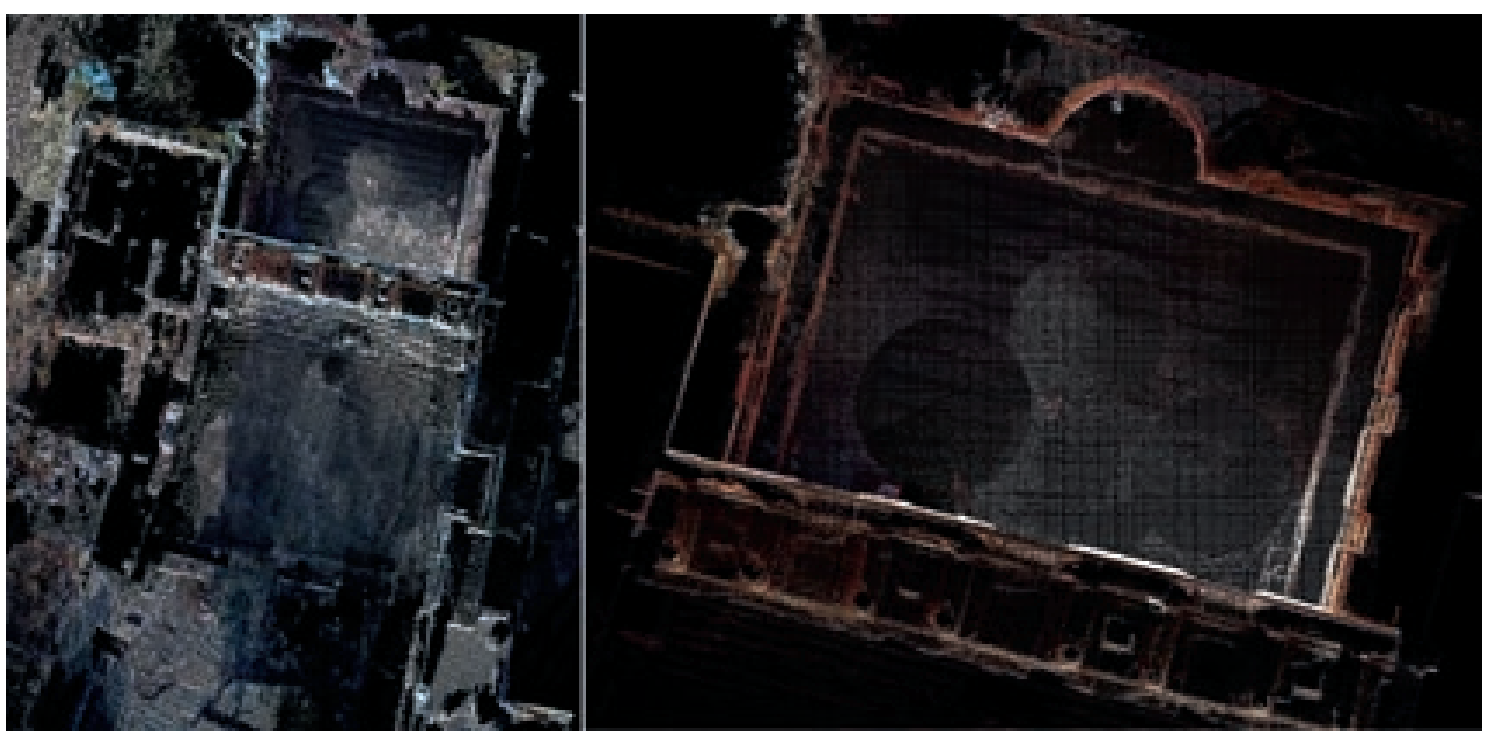

11 Grundrißpläne der Celsusbibliothek, erstellt aus den Scannerdaten

Architekturteilen und Gebäuden, für Detailbereiche an der Fassade der Celsusbibliothek wäre jedoch eine noch höhere Auflösung wünschenswert gewesen. Die dreidimensionale Datengrundlage läßt sich auf unterschiedliche Arten für verschiedene Anforderungen weiterverarbeiten, beispielsweise in Form eines detaillierten Grundrißplanes (Abb. 11).

Eine Aufnahme des gesamten Stadtgebietes von Ephesos scheint mit der getesteten Technologie ohne weiteres in vertretbarer Zeit möglich und könnte eine hochauflösende Grundlage für weitere wissenschaftliche oder Planungsaufgaben darstellen, wobei es aber sicher notwendig sein wird, entsprechende Strategien für systematische Messungen zu entwickeln.

\section{Ausblick}

Die derzeit aktuellen Modelle der 3-D-Laserscanner bieten bereits eine deutlich höhere Genauigkeit der Distanzmessung von $12 \mathrm{~mm}$ für einfache Scans und bis zu $6 \mathrm{~mm}$ bei mehreren gemittelten Scans. Der vergrößerte Scanbereich beträgt bei diesen Geräten $90^{\circ} \times 360^{\circ}$, sie weisen also keinen toten Winkel mehr auf. Das bedeutet, daß etwa in Innenräumen ein einziger Scan ausreichend ist. Die Geräte können fokussiert und dadurch besser für Vermessungen im Nahbereich eingesetzt werden. Eine weitere, für die archäologische Anwendung äußerst wichtige Entwicklung ist die direkte Koppelung des Scanners mit einer digitalen Kamera, so daß hochauflösende digitale Bilder in Verbindung mit der Objektgeometrie aufgenommen werden können. Dies macht den 3-D-Laserscanner zu einem wesentlichen Werkzeug für eine dreidimensionale Dokumentation von Ausgrabungen und historischen Monumenten. Die Entwicklung der Auswertungssoftware steht derzeit zwar noch am Beginn, die aktuellen Entwicklungen versprechen jedoch einen effizienten Einsatz in der Archäologie.

Vor weniger als fünf Jahren erschien es unvorstellbar, Gebäudestrukturen detailliert dreidimensional im Untergrund zu lokalisieren und als Ausgangsbasis für eine umfassende Interpretation, in diesem Fall des Stadtgebietes von Ephesos, zu nutzen. Die technischen Möglichkeiten des 3-D-Laserscanners bieten gemeinsam mit geophysikalischen Prospektionen gerade für eine Problematik wie die Erforschung dieser Stadt eine geeignete Ausgangsbasis: bekannte und ausgegrabene Reste können mit den noch im Untergrund verborgenen verbunden und unsere Vorstellungen dadurch beträchtlich erweitert werden. Schon die bisher erfaßten dreidimensionalen Daten mit allen ihren Einschränkungen bieten eine gute Grundlage für anschließende archäolo- 
gische Auswertung in Abhängigkeit der jeweiligen Fragestellungen. 3-D-Laserscanner haben sich in dem Testeinsatz als nutzbringendes Werkzeug erwiesen, das bald genauso gut in der Hand liegen sollte wie ein Spaten, eine Kelle oder der Bleistift.

Doz. Dr. Stefan Groh

Österreichisches Archäologisches Institut, Franz Klein-Gasse 1, A-1190 Wien

E-Mail: stefan.groh@oeai.at

Dr. Wolfgang Neubauer

VIAS - Vienna Institute for Archaeological Science, Franz Klein-Gasse 1, A-1190 Wien

E-Mail:wolfgang.neubauer@univie.ac.at

Abbildungsnachweis: alle Abb. C ÖAI. 